\title{
Retroperitoneal bronchogenic cyst: prenatal diagnosis of cystoid formation, its progression and surgery
}

\author{
Maly $\mathrm{T}^{1}$, Mihal $\mathrm{V}^{2}$, Michalkova $\mathrm{K}^{3}$, Tichy $\mathrm{T}^{4}$, Neoral $\mathrm{C}^{1}$, Zonca $\mathrm{P}^{5}$ \\ Department of Surgery, Palacki University Olomouc, Czech Republic
}

\begin{abstract}
Aim: Presentation of a rare localization of bronchogenic cyst in retroperitoneum.
Material: A case of a patient with retroperitoneal localization of a bronchogenic cyst with a prenatally diagnosed cystic formation. The surgery was indicated at the age of 6 owing to the progression of lesion. The histopathological examination of removed cyst revealed the diagnosis of bronchogenic cyst. For four years following the surgery, the patient was clinically free of complications. The regularly performed ultrasound examinations of the abdomen have been showing normal findings.

Conclusion: Despite the fact that retroperitoneal localization of bronchial cyst is very rare it should be considered in differential diagnosis (Fig. 3, Ref. 16). Text in PDF www.elis.sk.

Key words: retroperitoneal bronchogenic cyst, prenatal diagnosis, cystoid formation, progression and surgery.
\end{abstract}

The retroperitoneal bronchogenic cysts are a very rare finding. According to Pubmed, only 46 cases have been described in adults and children. Bronchogenic cysts are developmental abnormalities of primitive foregut. They develop between the third and sixth week of the embryonic development (1). They typically occur in lungs or mediastinum.

We are presenting an interesting case of a nine-year-old girl with prenatal diagnosis of a retroperitoneal cystoid formation, and its subsequent progress leading to surgery at the age of six. The further follow-up after the surgery shows well-being of the patient who has been completely free of complications.

\section{Case report}

The cystic lesion was prenatally diagnosed in the left upper quadrant of abdominal cavity in a female fetus. It was interpreted as a pancreatic cyst. After her delivery, neither clinical symptoms nor pathological findings were present in blood examination results. The follow-up took place in a surgical pediatric out-patient clinic based on confirmed diagnosis of congenital multilocular pancreatic cyst. Regular ultrasound examinations showed a slight progression of lesion. The CT scan was performed at the age of 3. Further observation was indicated. When the patient was 6 years old, MRI confirmed the progression of lesion as well (Fig. 1).

\footnotetext{
${ }^{1}$ Department of Surgery, Palacki University Olomouc, Czech Republic, ${ }^{2}$ Department of Pediatry, Palacki University Olomouc, Czech Republic, ${ }^{3}$ Department of Radiology, Palacki University Olomouc, Czech Republic, ${ }^{4}$ Department of Pathology, Palacki University Olomouc, Czech Republic, and ${ }^{5}$ Department of Surgery, University Ostrava, Czech Republic
}

Address for correspondence: T. Maly, Department of Surgery, Palacki University Olomouc, Czech Republic.
The formation in size of $15 \times 5 \times 5 \mathrm{~cm}$ in the retroperitoneal space dorsal from pancreatic corpus and tail was shown. It was suspected that the origin of the trilocular cyst was in the pancreatic tail. The six-year-old girl was clinically without any problems with normal digestive functions. A surgery was considered. After discussing the surgical intervention with the girl's parents, the operation was indicated.

During admission to hospital, the clinical examination revealed normal abdominal findings with no palpable mass. The general examination including lymphnodal status was normal. The girl was eutrophic. The surgery was performed under general anesthesia through midline laparotomy. The exploration of bursa omentalis was performed. A trilocular cyst complying with MRI finding was identified. The mass was located in retroperitoneum. The splenic artery and vein were dislocated by the cyst. The splenic flexure was mobilized. A complete separation of the cyst from the pancreas, splenic vessels, and spleen was performed. No communication with the pancreatic duct was found. The cyst was completely removed (Fig. 2). The surgery and post-operative course were free of complications. The drainage fluid contained increased levels of lipase and amylase immediately after the surgery. The blood levels of lipase and amylase were normal. The levels of lipase and amylase in the drainage fluid had returned to normal two days later and the drains were removed. The patient was discharged eight days after the surgery. The histopathological examination revealed a surprising diagnosis of bronchogenic cyst. The wall of the bronchogenic cyst contained a mature cartilage structure and mixed glands. The ciliated columnar epithelium and groups of alveoli with eosinophilic fluid were identified in the cysts (Fig. 3). The next several follow-ups performed up to the patient's current age have been uneventful. Regularly performed ultrasound examinations of the abdomen have shown no abnormality. The patient has been clinically free of complications. 


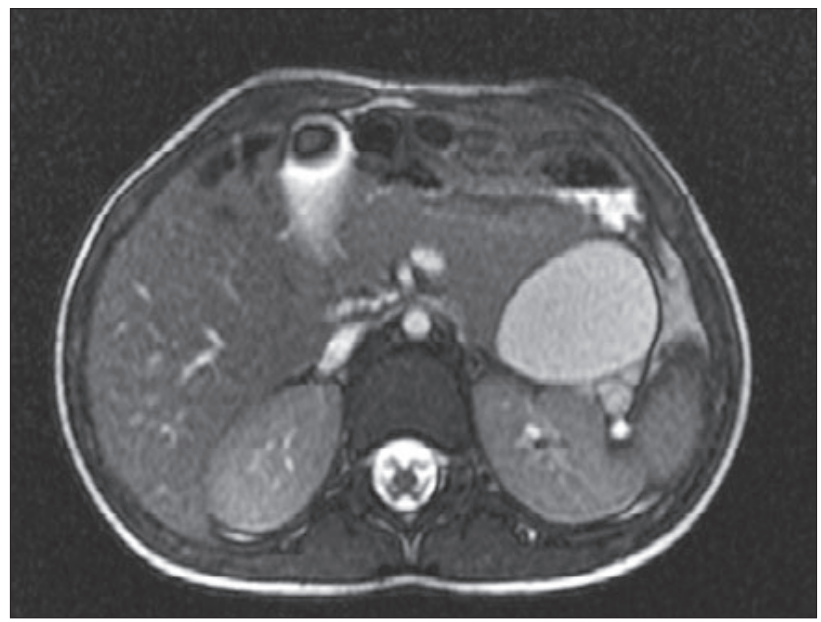

Fig. 1. MRI of abdomen, t2 trufi TRA sequence: Hyperintensive cystic formation in the left mesogastrium with the origin in pancreatic tail.

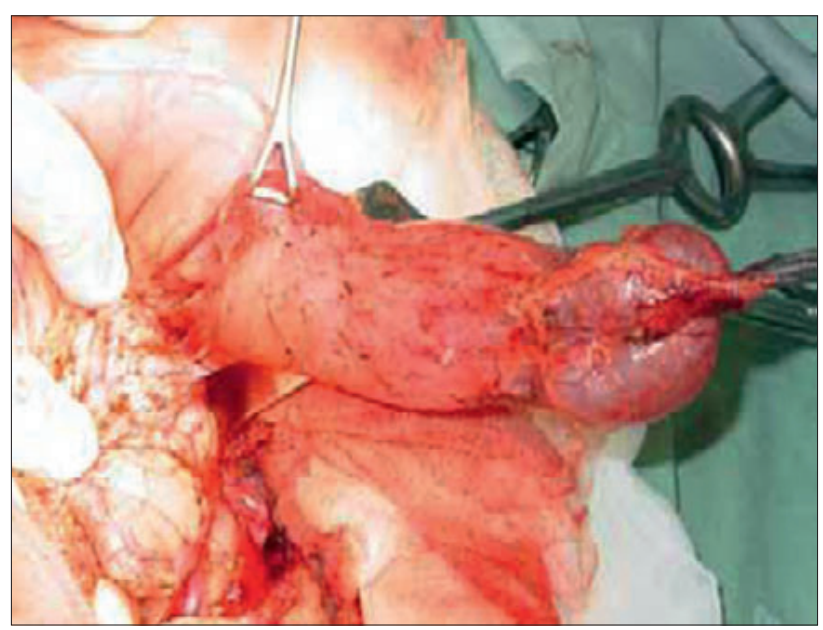

Fig. 2. Intra-operative picture: retropancreatic trilocular bronchogenic cyst before its separation from the dorsal pancreatic wall.

\section{Discussion}

Bronchogenic cysts are congenital developmental abnormalities of the primary ventral gut resulting from abnormal budding of the developing tracheobronchial tree with buds being separated from the normal airways $(1,2)$. According to literature, the prevalence of bronchogenic cysts ranges from 1/42.000 to 1/68.000 of population (3). They predominantly occur in men, namely in ratio of $4: 1$ (men : women) (4). They are typically localized in the supradiaphragmatic space, namely in lung parenchyma and dorsal mediastinum, particularly posterior to the carina or along the right trachea wall. Ectopic localizations such as lingual, supraclavicular, diaphragmatic, intrapericardial, spinal, abdominal, and in the neck have been described. The subdiaphragmatic location is rare $(5,6,7,8,9,10)$. The majority of these cysts $(82 \%)$ are located in an imaginary triangle envisioned posterior to the stomach, defined by the midline, splenic vein inferiorly, and spleen/diaphragm superiorly/posteriorly (11). Most of the cysts remain asymptom-

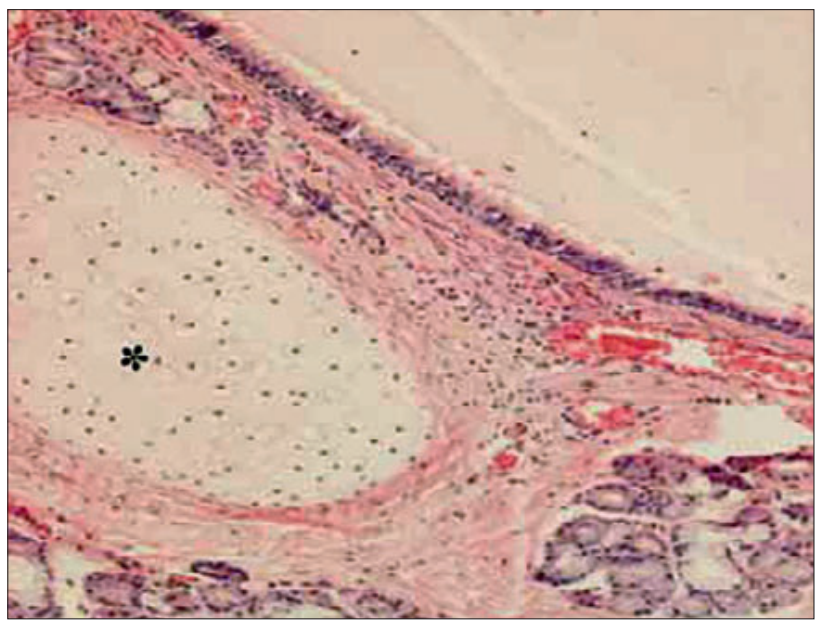

Fig. 3. The wall of bronchogenic cyst with mature cartilage $(*)$ and mixture glands. Ciliated columnar epithelium (hematoxylin-eosin, magnification 100x).

atic in patients. The clinical symptomatology occurs as a result of secondary infection, bleeding, perforation or compression of adjacent organs. The bronchogenic cyst in its typical localization could manifest itself through chest pain in the retrosternal area, dysphagia, cough, fever, and hemoptysis. The natural history of these cysts is unknown but the risk of malignancy is probably low (12). Malignant transformation of the cyst was described $(13,14)$. The diagnosis of a cyst localized in infradiaphragmatic space is usually coincidentally discovered when abdominal ultrasound or $\mathrm{CT}$ examination is performed for various other reasons. The differential diagnosis represents a wide scale of diseases. According to Yang, cystic lesions in the retroperitoneum can be classified as either neoplastic or non-neoplastic. Neoplastic lesions include cystic lymphangioma, mucinous cystadenoma, cystic teratoma, cystic mesothelioma, müllerian cyst, epidermoid cyst, tailgut cyst, bronchogenic cyst, cystic change in solid neoplasms, pseudomyxoma retroperitonei, and perianal mucinous carcinoma. Non-neoplastic lesions include pancreatic pseudocyst, non-pancreatic pseudocyst, lymphocele, urinoma, and hematoma. CT may provide important information about the lesion in respect of its location, size, and shape. In addition, it may reveal the involvement of adjacent structures, presence and thickness of its wall, as well as presence of septa, calcifications, or fat. In combination with clinical information, the relevant radiologic features allow adequate lesion characterization and diagnosis. The markers of non-invasiveness are important as they differentiate the character of retroperitoneal masses. It helps to provide a therapeutic strategy for patients (15). The exact diagnosis can be performed by histopathologicall examination after excision. Nevertheless, it is possible to diagnose these cysts preoperatively by EUS-FNA. To the best of our knowledge, the cases with prenatal diagnosis of cystoid formation, as described in our case, are extremely rare. The treatment of retroperitoneal bronchogenic cyst lies in its surgical removal. The excision is recommended in order to establish the diagnosis, alleviate any of symptoms, and prevent complications. The poten- 
$98-100$

tial of malignant transformation should be also considered when indicating for surgery. Obando suggested that surgery may not be necessary after the diagnosis of a retroperitoneal bronchogenic cyst by EUS-FNA (16).

\section{Conclusion}

Our case shows a rare prenatal diagnosis of a cystic lesion that was originally interpreted as a pancreatic cyst. A surgical removal was indicated because of lesion's progression. The histopathological examination revealed the diagnosis of a bronchogenic cyst. The retroperitoneal localizations of bronchogenic cysts are very rare but should be considered in differential diagnosis.

\section{References}

1. Siegelman ES, Birnbaum BA, Rosato EF. Bronchogenic cyst appearing as a retroperitoneal mass. AJR Am J Roentgenol 1998; 171: $527-528$

2. McCrystal DJ, Borzi PA. Retroperitoneoscopic resection of retroperitoneal bronchogenic cysts. Pediatr Surg Int 2002; 18: 375-377.

3. Sanli A, Onen A, Ceylan $\mathbf{E}$ et al. A case of a bronchogenic cyst in a rare location. Ann Thorac Surg 2004; 77: 1093-1094.

4. Pujary K, Pujary P, Shetty R et al. Congenital cervical bronchogenic cyst. Intern J Ped Otorhinolaryngol 2001; 57: 145-148.

5. Braffman B, Rhona K, Ellen SG et al. Subdiaphragmatic bronchogenic cyst with gastric communication. Gastrointest Radiol 1988; 13 : 309-311.
6. Haddadin WJ, Reid R, Jindal RM. A retroperitoneal bronchogenic cyst: a rare cause of a mass in the adrenal region. J Clin Pathol 2001; 54: 801-802.

7. Itoh H, Shitamura T, Katanka H et al. Retroperitoneal bronchogenic cyst: report of a case and literature review. Pathol Int 1999; 49: 152-155.

8. Matsubayashi J, Ishida T, Ozawa T et al. Subphrenic bronchopulmonary foregut malformation with pulmonary-sequestration-like features. Pathol Int 2003; 53: 313-316.

9. Murakami R, Machina M, Kobayashi et al. Retroperitoneal bronchogenic cyst: CT and MR imaging. Abdom Imaging 2000; 25: 444-447.

10. Paik SS, Jang KS, Han HX et al. Retroperitoneal bronchogenic cyst mimicking pancreatic pseudocyst in a patient with colorectal cancer. J Gastroenterol Hepatol 2005; 20: 802-803.

11. Liang MK, Yee HT, Song JW et al. Subdiaphragmatic bronchogenic cysts: a comprehensive review of the literature. Am Surg 2005; 71 : 1034-1041.

12. Murakami R, Machida M, Kobayashi Y, Ogura J, Ichikawa T, Kumazaki T. Retroperitoneal bronchogenic cyst: CT and MR imaging. Abdom Imaging 2000; 25: 444-447.

13. McCrystal DJ, Borzi PA. Retroperitoneoscopic resection of retroperitoneal bronchogenic cysts. Pediatr Surg Int 2002; 18: 375-377.

14. Sullivan SM, Okada S, Kudo M, Ebihara Y. A retroperitoneal bronchogenic cyst with malignant change. Pathol Int 1999; 49: 338-341.

15. Yang DM, Jung DH, Kim H, Kang JH, Kim SH, Kim JH, Hwang HY. Retroperitoneal cystic masses: CT, clinical, and pathologic findings and literature review. Radiographics 2004; 24 (5): 1353-1365.

16. Obando J, Merkle E, Bean SM. A Retroperitoneal Bronchogenic Cyst Clin Gastroenterol Hepatol 2009; 7 (8): A24-e1.

Received February 23, 2012. Accepted October 27, 2013. 